\title{
Codes for Iterative Decoding from Partial Geometries
}

\author{
Sarah J. Johnson \\ School of Elec. Eng. \& Comp. Sci. \\ University of Newcastle, \\ Callaghan NSW 2308, Australia \\ e-mail: sarah@ee.newcastle.edu.au
}

\begin{abstract}
This work develops codes suitable for iterative decoding using the sum-product algorithm. We consider regular low-density parity-check (LDPC) codes derived from partial geometries, a large class of combinatorial structures which include several of the previously proposed algebraic constructions for LDPC codes as special cases. We derive bounds on minimum distance and $\operatorname{rank}_{2}(H)$ for codes from partial geometries, and present constructions and performance results for two classes of partial geometries which have not previously been proposed for use with iterative decoding.
\end{abstract}

\section{INCIDENCE AND DESIGNS}

A design $\mathcal{D}=(\mathcal{P}, \mathcal{B}, \mathcal{I})$ consists of a finite non-empty set $\mathcal{P}$ of points and a finite non-empty set $\mathcal{B}$ of blocks, together with an incidence relation $\mathcal{I} \subseteq \mathcal{P} \times \mathcal{B}$. The incidence matrix $N$ of $\mathcal{D}$ is a $|\mathcal{P}| \times|\mathcal{B}|$ matrix indexed by the points and blocks of $\mathcal{D}$, with $N_{i, j}=1$ if point $i$ is incident with block $j$. The adjacency matrix of $\mathcal{D}$ is a $|\mathcal{P}| \times|\mathcal{P}|$ matrix $A$, indexed by the points of $\mathcal{D}$ with $A_{i, j}=1$ if the points $i$ and $j$ are incident with the same block in $\mathcal{D}$. The adjacency matrix is said to be strongly regular if the number of other points with which a pair of points are both incident depends only on whether or not the pair of points are themselves incident.

In what follows we consider partial geometries, a class of designs with strongly regular $A$. For a partial geometry, $\operatorname{pg}(s, t, \alpha)$, each point $p$ is incident with $t+1$ blocks and each block $B$ incident with $s+1$ points, any two blocks have at most one point in common, and for any non-incident pointblock pair $(p, B)$ the number of blocks incident with both $p$ and a point in $B$ equals some constant $\alpha$.

\section{CODES FRom PARTIAL GeOMETRIES}

We can take the incidence matrix $N$ of a partial geometry as the parity-check matrix $H$ of an LDPC code $C$ with $|\mathcal{P}|$ parity-checks, length $|\mathcal{B}|$, column weight $s+1$, row weight $t+1$, and girth $\geq 6$. Using the properties of strongly regular graphs we can find the eigenvalues of $A$ and then show that $N N^{T}$ has eigenvalues $(s+1)(t+1), s+t+1-\alpha, 0$ with corresponding multiplicities

$$
1, \frac{s t(s+1)(t+1)}{\alpha(s+t+1-\alpha)}, \frac{s(s+1-\alpha)(s t+\alpha)}{\alpha(s+t+1-\alpha)} .
$$

Using Tanner's bit- and parity-oriented bounds [3] we obtain the following

Lemma 1 The minimum distance of a code from $p g(s, t, \alpha)$ satisfies $d_{\min } \geq \max \{(t+1)(s+1-t+\alpha) / \alpha, 2(s+\alpha) / \alpha\}$.

Designs from two classes of partial geometries, balanced incomplete block designs, BIBDs $(\alpha=s+1)$, and generalized quadrangles, GQs $(\alpha=1)$, have been studied previously for
Steven R. Weller

School of Elec. Eng. \& Comp. Sci.

University of Newcastle, Callaghan NSW 2308, Australia

e-mail: steve@ee.newcastle.edu.au

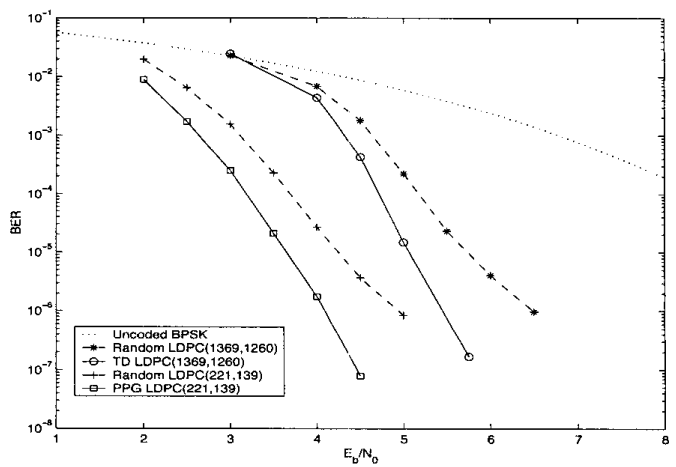

Figure 1: BER vs. $E_{b} / N_{0}$ for LDPC codes in an AWGN channel

use as LDPC codes (see e.g. [2, 4]). The minimum distance bounds from Lemma 1 are weak for the BIBDs, nets $(\alpha=t)$ and transversal designs, TDs $(\alpha=s)$, and a better bound is provided by Massey's bound ( $d_{\min } \geq s+2$ in this case). However, for the proper partial geometry $(1<\alpha<\min \{s, t\})$ and generalized quadrangle codes the bounds from Lemma 1 significantly improve upon Massey's bound to give a minimum distance bound of up to twice the column weight of $H$.

The excellent performance of the finite geometry codes [2] has been attributed to the highly redundant parity-check matrices of those codes [2, 4], motivating the search for other designs which give low rank parity-check matrices. A simple upper bound on the 2-rank of a code is the number of non-zero eigenvalues of $H H^{T}$ which we know from (1):

$$
\operatorname{rank}_{2}(H) \leq \frac{s t(s+1)(t+1)}{\alpha(t+s+1-\alpha)}+1
$$

Further, we use results from [1] to show that this bound is tight (within 1 of the actual rank) for the partial geometry codes with $\mu_{2}=s+t+1-\alpha \equiv 1 \bmod 2$. We see that, with the exception of some BIBDs, every partial geometry produces a code with linearly dependent rows in $H$.

Fig. 1 shows the performance of LDPC codes derived from a $\operatorname{TD}(2,36,2)$ and a proper $\operatorname{pg}(12,12,9)$ compared with randomly constructed codes of the same rate and length.

\section{REFERENCES}

[1] A. E. Brouwer and C. A. van Eijl. On the p-rank of strongly regular graphs. Algebra and Combinatorics, 1:72-82, April 1992.

[2] Y. Kou, S. Lin, and M. P. C. Fossorier. Low-density paritycheck codes based on finite geometries: A rediscovery and new results. IEEE Trans. Inform. Theory, 47(7):2711-2736, November 2001.

[3] R. M. Tanner. Minimum distance bounds by graph analysis. IEEE Trans. Inform. Theory, 47(2):808-821, February 2001.

[4] P. O. Vontobel and R. M. Tanner. Construction of codes based on finite generalized quadrangles for iterative decoding. In Proc. ISIT Washington, DC, June 24-29 2001. 